International Journal of Advanced Mathematical Sciences, 5(1) (2017) 8-19
International Journal of Advanced Mathematical Sciences
Website: www.sciencepubco.com/index.php/IJAMS
doi: $10.14419 /$ ijams.vil. 7128
Research paper

\title{
Machine-coded genetic operators and their performances in floating-point genetic algorithms
}

\author{
Mehmet Hakan Satman $^{1 *}$ and Emre Akadal ${ }^{2}$ \\ ${ }^{1}$ Istanbul University, Department of Econometrics \\ ${ }^{2}$ Istanbul University, Department of Informatics \\ *Corresponding author E-mail: mhsatman@istanbul.edu.tr
}

\begin{abstract}
Machine-coded genetic algorithms (MCGAs) use the byte representation of floating-point numbers which are encoded in the computer memory. Use of the byte alphabet makes classical crossover operators directly applicable in the floating-point genetic algorithms. Since effect of the byte-based mutation operator depends on the location of the mutated byte, the byte-based mutation operator mimics the functionality of its binary counterpart. In this paper, we extend the MCGA by developing new type of byte-based genetic operators including a random mutation and a random dynamic mutation operator. We perform a simulation study to compare the performances of the byte-based operators with the classical FPGA operators using a set of test functions. The prepared software package, which is freely available for downloading, is used for the simulations. It is shown that the byte-based genetic search obtains precise results by carrying out the both exploration and exploitation tasks by discovering new fields of the search space and performing a local fine-tuning. It is also shown that the introduced byte-based operators improve the search capabilities of FPGAs by means of convergence rate and precision even if the decision variables are in larger domains.
\end{abstract}

Keywords: Genetic algorithms; Optimization; Simulations

\section{Introduction}

Genetic Algorithms (GAs) are search and optimization methods that mimic the natural selection and the principles of genetics $[16,10]$. Since a $G A$ perform a selection mechanism on fitness values of candidate solutions rather than the goal function itself, it does not require the goal function to be neither continuous nor differentiable. This flexibility of GAs always opens new research areas, for instance, in Human-based GAs, there is not a goal function at hand and the human judgment is used to rank candidate solutions for the selection mechanism $[4,18,17]$.

Classical GAs perform an optimization process on an initially predefined or random population of solutions encoded using the binary alphabet. The selection operator is then applied to construct a mating-pool which is then used to re-combine candidate solutions with higher fitness values. A fitness value is a measurement of how well a candidate solution satisfies the goal function and/or a candidate solution is whether feasible or not. The generated solutions after re-combination are copied into the next generation with or without a slight modification. It is expected that the candidate solutions that have higher fitness values will then construct a better population after recombination and modification, namely, crossover and mutation, respectively.

It is natural to solve $0-1$ problems with the classical GAs. Since an integer can be formed up using bits that are sequenced in order, an optimization problem with integer decision variables are also natural by means of binary coding. However, representing real values is not possible because infinite number of bits are required. To cope with this issue, a sequence of moderate number of bits can be combined to map some integers to real numbers in a discreate manner. Assume that $b_{i}$ is an element of a binary chromosome and $Q$ is the mapped integer value. The corresponding real number $R$ mapped into the range $[A, B]$ can be obtained using the formula

$$
R=A+\frac{B-A}{2^{L}-1} \times Q
$$

where $L$ is the length of choromosome $b$. With using this formula, a conversion between binary strings and real numbers in a predefined range is possible. However, this process yields another kind of problems in $G A$ s, that is, as the bit strings get longer, longer number of iterations are required to obtain the global optima. Since the diversity of population is also important, candidate solutions with longer bit strings require the size of population to be higher to represent the different areas of the seach space $[19,11]$.

In FPGAs (Floating-point genetic algorithms), the candidate solutions are not encoded using a binary alphabet and the new solutions are recombined and altered using a different logic. The generated content is generally linear or a nonlinear function of the parents or drawn randomly in a pre-defined range. Mutation is performed by adding a single value which fallows a probability distribution with constant or dynamic parameters $[20,5]$. Since the classical recombination operators are defined on bit strings, new type of genetic reproduction operators were developed for the FPGA.

MCGAs (Machine-coded genetic algorithms) are an other type of $G A$ s which use the byte representation of real values in the computer memory. Compilers and interpreters encode numbers (includ- 
ing floating-point types) using constant size byte strings using a standard. Assuming these byte strings as the geno-type makes the classical crossover operators applicable on the candidate solutions which are vectors of real numbers. Since the mutation operator in $M C G A$ s is applied on the geno-type and the position of each single byte has a different effect on pheno-type, the byte-based mutation operator gains a similar interpretation with its binary counterpart. It is shown that the MCGAs outperform some other evolutionary algorithms for some cases in a recently published study [28]. In this paper, we introduce other types of byte-based genetic operators and show their performances using a simulation study. In Section 2 we give a brief introduction for FPGAs. In Section 3 we show the effects of the byte-based genetic operators that are recently developed. In Section 4 we develop new kind of byte-based genetic operators for improving the genetic search. In Section 5, we give a brief introduction for the developed software for MCGAs. In Section 6, we perform a simulation study on a set of test functions. Finally in Section 7 , we conclude.

\section{Floating-point genetic algorithms}

In genetic algorithms, individuals are encoded using a binary alphabet, mathematically $b_{i} \in\{0,1\}$, using an encoder function $r=d(b)$. The encoder function is invertible, that is, each single chromosome $b$ can be produced using the decoder function $b=d^{-1}(r)$, where $b$ is the binary chromosome, $r$ is the corresponding real number such that $r \in \mathbb{R}, d^{-1}($.$) is the inverse of the function d($.$) . If a chro-$ mosome $c$ is a vector of real values rather than binary digits, then the distinction between the encoded and the decoded values is disappeared. The genetic algorithms in which the chromosomes are directly used without encoding-decoding are called floating-point or real-valued genetic algorithms (FPGAs).

Classical crossover operators combine two binary chromosomes in many different ways. Since the chromosomes are real-values in $F P G A$ s, binary crossover operators are not directly applicable on a real vector. As the classical mutation operator simply flips a binary value of a chromosome, it is not even possible to make an analogy for a real vector. To cope with these issues, new crossover and mutation operators are developed for FPGAs. Note that the other operators such as selection and elitism are not related to reproduction operators as they are only based on the fitness or cost values.

Suppose that the chromosomes $A$ and $B$ have real values $a_{1}, a_{2}, \ldots$, $a_{p}$ and $b_{1}, b_{2}, \ldots, b_{p}$ where $p$ is the chromosome length or the number of real values. The Flat Crossover [15, 25] operator produces two offspring $C$ and $D$ such that

$$
C=\left(c_{1}, c_{2}, \ldots, c_{p}\right)
$$

and

$$
D=\left(d_{1}, d_{2}, \ldots, d_{p}\right)
$$

where $c_{i}$ and $d_{i}$ are random variables which follow a Uniform distribution with parameters $\min \left(a_{i}, b_{i}\right)$ and $\max \left(a_{i}, b_{i}\right)$, and $i=1,2, \ldots p$. This operator serves a natural way of generating values in their definition ranges.

Arithmetic Crossover [20] generates two offsping $C$ and $D$ such that

$$
c_{i}=\alpha a_{i}+(1-\alpha) b_{i}
$$

and

$$
d_{i}=(1-\alpha) a_{i}+\alpha b_{i}
$$

where $\alpha$ is a random variable that follows a Uniform $(0,1)$ distribution. $\alpha$ can also be selected adaptively by changing its value depending on the current number of generations. The generated variables are simply weighted means of the variables of parents. This operator also ensures the bounds of variables naturally.
$B L X-\alpha$ Crossover $[8,14]$ is an other type of crossover operators and generates the components $c_{i}$ and $d_{i}$ of chromosomes $C$ and $D$ randomly using a Uniform distribution with parameters $r_{\text {min }}-I \alpha$ and $r_{\text {max }}+I \alpha$ where $r_{\text {min }}=\min \left(a_{i}, b_{i}\right), r_{\text {max }}=\max \left(a_{i}, b_{i}\right)$, and $I=r_{\max }-r_{\min }$. If the $\alpha$ parameter is selected as $\alpha=0$ then the operator generates offspring randomly within the bounds as the Flat Crossover does. As the parent chromosomes $A$ and $B$ stay in the different areas of the solution space, the value of $I$ increases. Since larger values of $I$ expand the range of parameters of the Uniform distribution, the chance of obtaining diverse solutions increases. $\alpha$ determines the magnitude of this diversity. As the parent chromosomes get close and lay on a similar subspace of the solutions, the generated offspring get closer to parents. This operator does not ensure the boundary constraints to be satisfied.

Linear Crossover [35, 15] works in a different way as it performs an inner selection mechanism between generated offspring. The generated offspring $J_{1}, J_{2}$, and $J_{3}$ are defined as

$$
\begin{aligned}
J_{1} & =\frac{1}{2} A+\frac{1}{2} B \\
J_{2} & =\frac{3}{2} A-\frac{1}{2} B \\
J_{3} & =-\frac{1}{2} A+\frac{3}{2} B
\end{aligned}
$$

where $J_{1}=\left(j_{11}, j_{12}, \ldots, j_{1 p}\right), J_{2}=\left(j_{21}, j_{22}, \ldots, j_{2 p}\right)$, and $J_{3}=$ $\left(j_{31}, j_{32}, \ldots, j_{3 p}\right)$, respectively. The operator generates 3 offspring, rather than 2 , and returns a vector of best two as the result. This operator may generate offspring out of the variable bounds.

Simulated Binary Crossover (SBX) simulates the single-point crossover that used in binary genetic algorithms for the realparameters $[6,7]$. Now suppose that $\beta_{i}$ is a random variable which is defined as

$$
\beta_{i}=\frac{d_{i}-c_{i}}{b_{i}-a_{i}}
$$

and has a probability density function

$$
P\left(\beta_{i}\right)= \begin{cases}0.5\left(\eta_{c}+1\right) \beta_{i}^{\eta_{c}} & , \beta_{i} \leq 1 \\ 0.5\left(\eta_{c}+1\right) \frac{1}{\beta_{i}^{\eta_{c}+2}} & , \beta_{i}>1\end{cases}
$$

where $\eta_{c}$ is a positive real number. If $\eta_{c}$ is a small number, generated offspring will stay far away from the parents. This constant can be selected from the range of $2 \leq \eta_{c} \leq 5$ [5].

Now suppose that $\beta_{q_{i}}$ is a random number that is drawn using the probability density function $P\left(\beta_{i}\right)$. The operator generates two offspring using formulas

$$
c_{i}=0.5\left[\left(1+\beta_{q_{i}}\right) a_{i}+\left(1-\beta_{q_{i}}\right) b_{i}\right]
$$

and

$$
d_{i}=0.5\left[\left(1-\beta_{q_{i}}\right) a_{i}+\left(1+\beta_{q_{i}}\right) b_{i}\right]
$$

where $a_{i}$ and $b_{i}$ are real parameters of parent vectors $A$ and $B$, respectively. Drawing a $\beta_{q_{i}}$ randomly using its probability density function can be performed using the Probability Integral Transformation. Now suppose that $u$ is a random variable that follows a Uniform $(0,1)$ distribution. Then the random variable $w$ which satisfies the relation

$$
u=\int_{-\infty}^{w} \varphi(x) d x
$$

follows a $\varphi$ distribution. Analogically, 
$\beta_{q_{i}}= \begin{cases}(2 u)^{\frac{1}{\eta_{c}+1}} & , u \leq 0.5 \\ \left(\frac{1}{2(1-u)}\right)^{\frac{1}{\eta_{c}+1}} & , u>0.5\end{cases}$

follows a $P\left(\beta_{i}\right)$ distribution. Using this cumulative distribution function, randomly simulated $\beta_{q_{i}}$ values can be obtained after drawing random numbers from the Uniform distribution.

Unfair Average Crossover [21] generates offspring $C$ and $D$ with components $c_{i}$ and $d_{i}$ using the formulas

$c_{i}= \begin{cases}(1+\alpha) a_{i}-\alpha b_{i} & , i=1, \ldots, j \\ -\alpha a_{i}+(1+\alpha) b_{i} & , i=j+1, \ldots, n\end{cases}$

and

$d_{i}= \begin{cases}(1-\alpha) a_{i}+\alpha b_{i} & , i=1, \ldots, j \\ \alpha a_{i}+(1-\alpha) b_{i} & , i=j+1, \ldots, n\end{cases}$

where $1 \leq j \leq n$ is the randomly selected cut-point, $\alpha$ is a random variable that follows a Uniform $(0,0.5)$ distribution. Since some weights are negative, this operator does not ensure the boundary constraints of variables to be satisfied.

As it is shown above, crossover operators generate offspring that random, linear or non-linear functions of parents, numerically. The crossover operators developed for FPGAs, do not mimic their binary counterparts as they are not performed on the basic elements of numbers, namely geno-type. Even though the FPGA recombination operators perform successful searches in many optimization problems, there is a need for some enhanced operators, at least in some cases. [13] stressed that the main problem in FPGAs is the balance of $e x$ ploration and exploitation and as a consequence, the problem of premature convergence. In addition to the selection of right amount of population size, maximum number of iterations, and number of elitist solutions; it is vital to select the right combination of genetic recombination operators which perform the tasks of exploration and exploitation.

\section{Machine-coded genetic operators}

Natural coding of human DNA consists of an alphabet $\S$ with four digits or elements, that is, $\S=\{A, T, C, G\}$. In an optimization problem, if the decision variables have only two values, the natural alphabet is $b=\{0,1\}$. For instance, in the Knapsack problem [23, 22], chromosomes are naturally bit strings and each single bit value is mapped to a decision variable. When the decision variables are in type of integer, more than one bits are combined to form up the value of an integer variable using encoder and decoder functions. Finally, presentation of real numbers is even possible in a discrete manner, but not continuously. Binary coding in classical genetic algorithms is the natural way of presenting variables and the classical reproduction operators such as crossover and mutation perform sensible tasks.

FPGAs are also genetic algorithms in which candidate solutions are not encoded using an alphabet and genetic operators are performed directly on them. Dropping encoding-decoding scheme makes the classical reproduction operators useless. Special reproduction operators developed for FPGAs combine parents linearly or non-linearly using arithmetic operations. However, real values in computer memory are already stored using byte values in a discrete manner but with a great level of precision which can be satisfied using longer chromosomes in classical GAs. The classical crossover operator can be directly applied on the byte representation of real values. Classical mutation operator can also be simulated with its byte-based counterpart.
Suppose that a variable with the type of double in C++ language has the value of 1234.56789 . This value is encoded in computer memory using the byte values in type of unsigned char

$$
b_{i}=(231,198,244,132,69,74,147,64)
$$

using the encoding standard IEEE 754 - (IEEE Standard for Floating-Point Arithmetic) $[9,31]$ where $0 \leq b_{i} \leq 255$ for $i=$ $1,2, \ldots, 8$. This coding scheme makes classical crossover operator applicable as the number is represented in an elementary way. Now suppose that an other double-typed value of 612347676.566 is encoded using the same standard as

$$
c_{i}=(176,114,72,142,215,63,194,65)
$$

where $0 \leq c_{i} \leq 255$ for $i=1,2, \ldots, 8$. Now the single-point, twopoint and uniform crossover operators are directly applicable on $b_{i}$ and $c_{i}$ as in the binary genetic algorithms. By selecting a random cut-point $k=4$, the one-point crossover generates two offspring $d_{i}$ and $e_{i}$ as

$$
\begin{gathered}
d_{i}=(231,198,244,132,215,63,194,65) \\
e_{i}=(176,114,72,142,69,74,147,64)
\end{gathered}
$$

which are the byte-representations of double-typed values 612347657.9123 and 1234.5679, respectively. As it is shown above, the offspring $d_{i}$ has a value very close to parent $c_{i}$. Addition to this, $e_{i}$ is almost equal to $b_{i}$ with a slight change. Table 1 shows all possible offspring values that one-point crossover operator can generate Note that, since the number of generated offspring is limited with the number of cut points, the uniform crossover can be selected for obtaining maximum number of unique offspring.

Table 1: One-point byte-crossover on two parents

\begin{tabular}{rrr}
\hline Cut-point & Offspring $_{1}$ & Offspring $_{2}$ \\
\hline 1 & 612347676.5660 & 1234.5679 \\
2 & 612347676.5686 & 1234.5679 \\
3 & 612347677.9123 & 1234.5679 \\
4 & 612347657.9123 & 1234.5679 \\
5 & 612272905.9123 & 1234.7105 \\
6 & 613714697.9123 & 1231.9605 \\
7 & 80908641.2390 & 9343.6840 \\
\hline
\end{tabular}

As mentioned above, byte-crossover operator serves a natural way of applying binary crossover operators on the real-valued chromosomes. The byte-mutation operator works in a way that similar to its binary counterpart. First, the operator selects a byte $b_{r}$ randomly with the probability of mutation $P_{m}$. Then a random value $u$ is selected using a Uniform $(0,1)$ distribution. If $u<0.5$ then the byte value $b_{r}$ is increased by 1 , otherwise, it is decreased by 1 [28]. If the mutated byte value $b_{r}^{*}$ is greater than the maximum byte value 255 , then it is altered as $b_{r}^{*}=0$. Similarly, if the mutated value is smaller than the minimum byte value 0 , then is is altered as $b_{r}^{*}=255$.

Table 2 shows the differential effect of a mutated byte on the value of 1234.56789. In the first row of Table 2, the most left byte is increased by 1 and a very small change is observed on the corresponding value. In the second row, it can be shown that the effect of the second byte is more clear but it is still small. After changing 7th byte value from 147 to 148 the integer part of the corresponding value changes from 1234 to 1298 . Finally, a change on the most right byte has the maximum effect and the resulted value has an integer part of 80908641 . The byte-mutation operator performs a similar task when it is compared to binary mutation operator as the difference between the original and the mutated value depends on the location of the mutated gene. The byte-coded mutation operator also performs local fine-tuning operations well in a wide range. 
Table 2: Effect of the byte-coded mutation operator

\begin{tabular}{rr}
\hline$b_{i}^{*}$ & Value \\
\hline$(\mathbf{2 3 2}, 198,244,132,69,74,147,64)$ & 1234.56789000000026135240 \\
$(231, \mathbf{1 9 9}, 244,132,69,74,147,64)$ & 1234.56789000005824163964 \\
$(231,198, \mathbf{2 4 5}, 132,69,74,147,64)$ & 1234.56789001490119517257 \\
$(231,198,244, \mathbf{1 3 3}, 69,74,147,64)$ & 1234.56789381469729960372 \\
$(231,198,244,132, \mathbf{7 0}, 74,147,64)$ & 1234.56886656250003397872 \\
$(231,198,244,132,69,75,147,64)$ & 1234.81789000000003397872 \\
$(231,198,244,132,69,74, \mathbf{1 4 8}, 64)$ & 1298.56789000000003397872 \\
$(231,198,244,132,69,74,147, \mathbf{6 5})$ & 80908641.2390400022268295 \\
$(231,198,244,132,69,74,147,64)$ & 1234.56789000000003397872 \\
$(231,198,244,132,69,74,147, \mathbf{6 3})$ & 0.01883801101684570364 \\
$(231,198,244,132,69,74, \mathbf{1 4 6}, 64)$ & 1170.56789000000003397872 \\
$(231,198,244,132,69,73,147,64)$ & 1234.31789000000003397872 \\
$(231,198,244,132, \mathbf{6 8}, 74,147,64)$ & 1234.56691343750003397872 \\
$(231,198,244, \mathbf{1 3 1}, 69,74,147,64)$ & 1234.56788618530276835372 \\
$(231,198, \mathbf{2 4 3}, 132,69,74,147,64)$ & 1234.56788998509887278487 \\
$(231, \mathbf{1 9 7}, 244,132,69,74,147,64)$ & 1234.56788999994182631781 \\
$(\mathbf{2 3 0}, 198,244,132,69,74,147,64)$ & 1234.56789000005801426596 \\
\hline
\end{tabular}

\section{Improving the Search Capabilities}

The byte based crossover and mutation operators that are mentioned in Section 3 outperform some other optimization techiques on some test functions as in reported in [28]. Addition to one-point crossover operator, the other well-known crossover operators such as twopoint crossover and uniform crossover can be implemented in a way similar to their binary counterparts.

Despite the crossover operator can be directly applied on the byterepresentations of candidate solutions, the byte-based mutation operator can be applied in several ways. In the previously reported and first case, a randomly selected byte is altered by adding a +1 or -1 with probabity $1 / 2$. This type of mutation changes the real value of chromosomes depending on the location of the mutated byte but more generations are required to achive a part of a solution space because the amount of alteration is constant and almost always equal to 1 .

In the second case, a randomly selected byte can be drawn randomly in the range of $0-255$, which is the natural range of byte representation. This operator serves a different approach to the binary mutation and can mimic the behaviour as the effect of mutation depends on the location of the mutated byte. As a result of this, the tasks of searching different areas of the solution space and performing the local fine-tuning is carried out by the operator in a more effective way when it is compared to the original byte-based mutation operator.

The two-variables Easom function is defined as

$$
f(x, y)=-\cos (x) \cos (y) \exp \left(-\left((x-\pi)^{2}+(y-\pi)^{2}\right)\right)
$$

for $-100 \leq x, y \leq 100$ and used to test the performances of some evolutionary algorithms $[2,3,27]$. The function has a global minimum of -1 for $x=\pi$ and $y=\pi$. Altough the function is smooth and has only two variables, many optimization methods including Hooke-Jeeves and Nelder-Mead fail to find the global minimum despite using many starting points. For a good starting point, for instance $(3.14,3.14)$, Nelder-Mead reports

$$
x=3.141591488522633923
$$

and

$$
y=3.141592522488581451
$$

for tolerance of $10^{-10}$ and maximum number of iterations $10^{6}$ where $\pi=3.141592653589793116$ with the same number of digits.
A genetic algorithm with byte-based uniform crossover and bytebased random mutation reports the solution as 1

$$
x=3.1415926516056060791
$$

and

$$
y=3.1415926665067672729
$$

which is more precise than the classical optimization method. $M C G A$ operators, unlike the reproduction operators in evolutionary algoritms in general, obtain a solution close to the real solution without using an external optimization techinique in terms of hybridization. In Figure 1, the best and the average fitness values of the genetic search is plotted by generations. It is shown that the byte-based operators perform the tasks of exploration and exploitation. The algorithm suddenly discovers a better solution in Generation $\approx 50$ and performs a local fine-tuning using this solution to obtain a solution closer to the real solution in following generations. Finally, the reported $f(x, y)$ value is almost equal to its minimum for the precise estimates of $x$ and $y$.

In Figure 1, it is also shown that the average fitness does not converge to the best solution by iterations. It is directly related to the mutation probability, that is, algorithm always alters solutions to get closer to the real solution by means of exploration. Alternatively, the mutation probability can be kept higher in very early stages of the genetic search and shrinked by generations and finally set to zero in last iteration. Now suppose that the mutation probability at generation $t$ is defined as

$$
P_{m}^{(t)}:=P_{m}-\frac{t}{T} \times P_{m}
$$

where $P_{m}$ is the initial mutation probability, $t$ is the current number of generations, and $T$ is the maximum number of generations. Using this formula, the mutation probability for the initial population is set to $P_{m}$ whereas it is 0 in the last generation when $t=T$. If the initial mutation probability $P_{m}$ is set to a higher value, then the task of exploration is more prominent than the task of exploitation because the algorithm tends to jump many different areas of the seach space frequently, however, as the $t$ increases by time, the $P_{m}(t)$ also decreases and the task of exploitation becomes more prominent and the population converges to the real solution. In Figure 2, convergence of the average fitness values is shown when the dynamic mutation probabilities are used instead. It is also shown in Figure 2

${ }^{1}$ Maximum number of generation $=500$, population size $=200$, selection method $=$ Tournament selection, mutation probability $=0.20$, crossover probability $=1.0$, number of elitist solutions $=2$ 


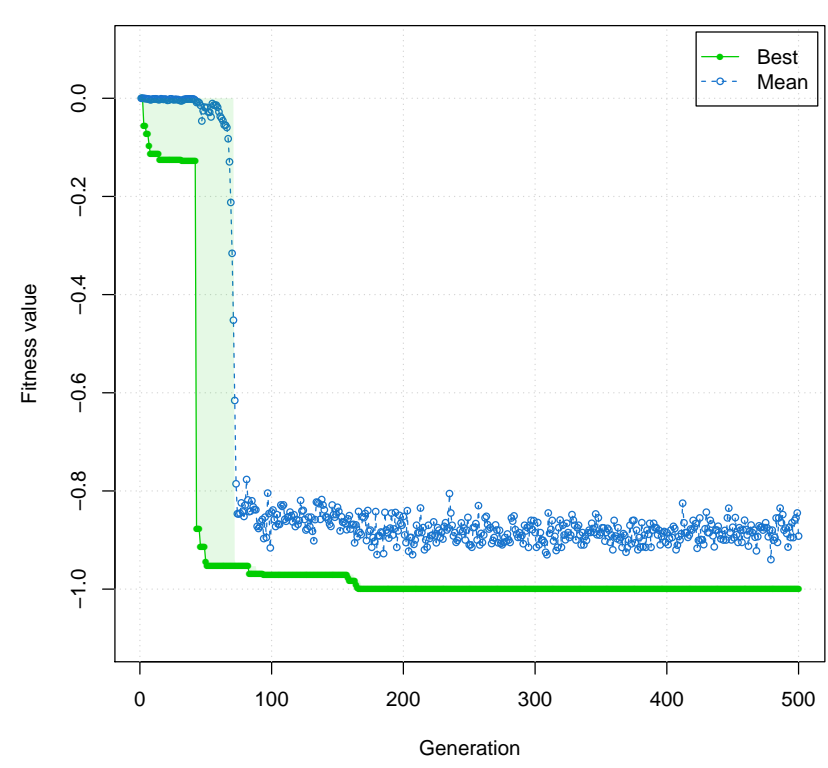

Figure 1: Minimum of Easom function by generations

that as the mutation probability decreases by iterations, the average fitness approaches to the best fitness, that is, not only a single candidate solution but the whole population converges.

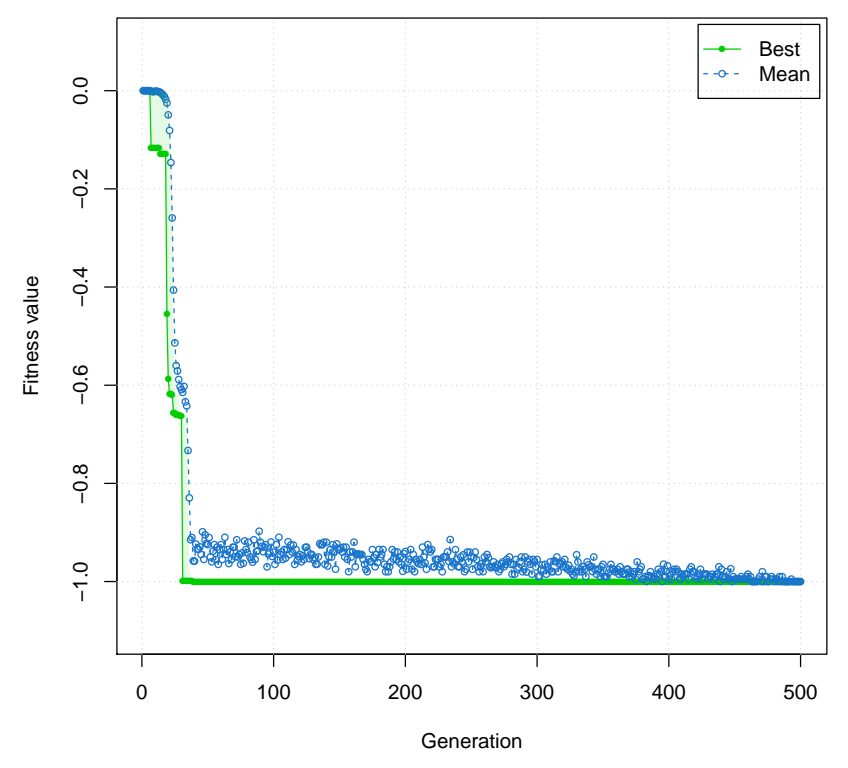

Figure 2: Minimum of Easom function by generations with dynamic mutation probabilities

The $n$-variable Rastrigin function is defined as

$$
f(\vec{x})=\sum_{i=1}^{n} x_{i}^{2}-10 \cos \left(2 \pi x_{i}\right)+10
$$

for

$$
-5.12 \leq x_{i} \in \vec{x} \leq 5.12
$$

and it is used in the simulation study in Section 6. The function has many local optima and it is a hard job to find to global minimum $f(\vec{x})=0$ for $\vec{x}=[0,0, \ldots, 0]^{T}$ even in a narrower domain. Since the original domain is $[-5.12,5.12]$ for all $x_{i}$ in $\vec{x}$, the function has finite number of local optima. When the domain is changed to $(-\infty, \infty)$, the function has infinite number of local optima and the problem of finding the global minimum gets more difficult. Table 3 summarizes the results of a mini simulation with a limited genetic search. The number of maximum iterations is set to 500 so the genetic search is prevented from finding the global minimum in later generations for comparison. The population size is set to 100 for all operators. This configuration is limited for such a function like Rastrigin with 50 variables with domains $\left[-10^{30}, 10^{30}\right]$, because, a moderate size of population and a limited number of generations may not be enough to represent different fields of the search space and lucky crossover and mutation operations may never come to pass to achieve the global minimum. In Table 3 , it is shown that the winners are byte-based (uniform) crossover, linear crossover, and unfair-average crossover when the succeeding criteria are both the achievement the global minimum and time efficiency. The genetic search with byte-based uniform crossover is run with the dynamic random mutation operator, whereas the others run with the random uniform mutation. Note that the values that are reported in Table 3 are machine and implementation dependent, that is, the order of magnitude of algorithms can differ from the time efficiency when the operators are implemented in different algorithms and run in different machines. Byte-based crossover and mutation operators also perform a bound check which can take additional time.

\section{Prepared Software}

We prepared an $\mathrm{R}$ [24] package, namely mcga, and it is freely available for downloading on $C R A N^{2}$. The package includes functions for byte-based genetic operators which can co-operate with the function ga of package GA [30]. mcga implements the byte-based genetic operators

- byte_crossover,

- byte_crossover_1p,

- byte_crossover_2p,

- byte_mutation,

- byte_mutation_dynamic,

- byte_mutation_random,

- byte_mutation_random_dynamic

for uniform crossover, one-point crossover, two-point crossover, byte-based \pm 1 mutation, byte-based \pm 1 mutation with dynamic mutation probabilities, byte-based random mutation, and byte-based random mutation with dynamic mutation probabilities, respectively. In ga function of package GA, user-defined crossover and mutation arguments can be set using the argument names crossover= and mutation=, respectively. For instance, a genetic search with uniform crossover and dynamic mutation requires an $\mathrm{R}$ function call like

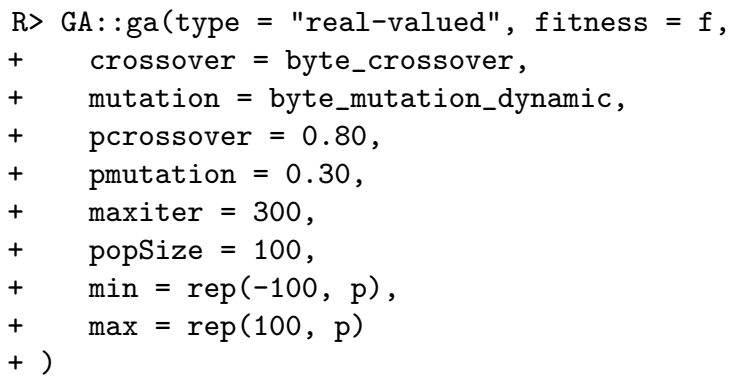

${ }^{2}$ The web page for the package is https://cran.r-project.org/web/ packages/mcga/index.html and it can be installed and loaded by the standard R functions install. packages and require. 
Table 3: 50-variables Rastrigin with domain $x_{i} \in\left(-10^{30}, 10^{30}\right)$

\begin{tabular}{|r|rrrrrrr|}
\hline & Byte & SBX & Flat & Arithmetic & BLX & Linear & Unfair \\
\hline Fitness & 0 & $>10^{56}$ & $>10^{57}$ & $>10^{57}$ & $>10^{56}$ & 0 & 0 \\
\hline Time & 13.23 & 19.11 & 28.41 & 12.20 & 34.59 & 10.44 & 10.8 \\
\hline Time\% & 100 & 144.44 & 214.73 & 92.21 & 261.45 & 78.91 & 81.63 \\
\hline
\end{tabular}

where "real-valued" is the string for type of the decision variables, $f$ is the goal function to be maximized, byte_crossover is the desired crossover function,

byte_mutation_dynamic is the desired mutation function, 0.80 is the crossover probability, 0.30 is the first value of the mutation probability which will be then decreased by iterations, 300 is the maximum number of iterations or generations, 100 is the population size, $\operatorname{rep}(-100, p)$ is the vector of lower bounds of the $p$-decision variables, and $\operatorname{rep}(100, p)$ is the vector of upper bounds of the $p$ decision variables, respectively. The byte-based reproduction operators listed above wraps all of the low-level byte operations that are written in the $\mathrm{C}++$ language. As a consequence, the goal function to be optimized is a simple $R$ function which takes a real vector as an argument. The defined recombination operators generate offspring when they are called during the optimization process and hide the low-level details from the user. This type of design also makes possible to develop and implement new kind of byte-based operators, possibly, in a future work.

\section{Simulation study}

We prepare a simulation study to reveal the performances of the byte-based crossover and mutation operators with the recently developed genetic operators that are developed for the FPGA. The simulation suite consists on the well-known test functions that are used for comparing the performances of the optimization algorithms and they are listed in Table 4 [12, 32, 34, 26, 1, 29, 33]. Since the test functions include summation and production operators, the number of parameters $p$ is variable and we set to $p=25, p=50$, and $p=75$. By using these scales, it is planned to measure the performances of the operators in different dimensions of the search spaces by means of tendency of failure respect to an increase on the number of parameters. Note that the genetic search tends to converge the global minimum of 0 of all functions in the test suite when the population size and the maximum number of generations are increased. We set the population size to 100 and the maximum number of generations to 200 for all configurations to make the comparison more clear, that is, a genetic search with different crossover and mutation operators has a different convergency rate in early stages of the optimization process. The crossover and the mutation probabilities are set to 0.80 and 0.10 , respectively. In each single generation, the best 5 solutions are directly copied into the next population without any ruining. Simulations are performed using the R software [24] with packages GA [30] and mcga [28] are installed. The simulation results are shown in Table 5, Table 6, and Table 7 for $p=25, p=50$, and $p=75$, respectively.

Table 5 summarizes the results for $p=25$. In Table 5 , functions are listed in the column 1 . For each single row, results are reported for the byte-based mutation operator and the FPGA mutation operator which is based on adding some random values on randomly selected variables. Columns $3-9$ shows the results for different kind of crossover operators. In Table 5, it is shown that in a genetic search with byte-based mutation and crossover operators, the average of the minimum value of Ackley function is reported as 0 , that is, performing genetic search on the Ackley function 250 times, we obtain 0 in average. It is also shown in the Table 5 that the bytebased crossover and the FPGA mutation operator results a higher average value 1.294 . Similarly, a genetic search with the byte-based operators result 0 in average, whereas the minimum is reported as
177.261 by the byte-based crossover and the FPGA mutation operator for the function Bohacevsky. The linear crossover operator is the winner and it works well with either standard and byte-based mutation operators. Finally, in total of $14 \times 7=98$ cases, the bytebased mutation operator exposes a better or equal performance in 85 cases when different kind of crossover operators are used. When the byte-based crossover and the byte-based mutation operators are combined together, the byte-based operators have higher or equal performance with the winner for the functions Ackley, Bohacevsky, Bolzman, Hyper-ellipsoid, Maxmod, Multimod, Rastrigin, Schaffer, Schwefel, Sphere, and Sumsquares. The byte-based operators are out-performed by the other operators in a single case in which the Levy function is used.

Table 6 shows the simulation results for $p=50$ and follows the same logic in representing the simulation results for $p=25$. The byte-based mutation operator exposes a better or equal performance in 77 out of 98 cases when different kind of crossover operators are used. The evarage of genetic searches have better or equal performances of the winner for the functions Ackley, Bohacevsky, Holzman, Hyperellipsoid, Multimod, Rastrigin, Schewel, Sphere, and Sumsuares. Differently, the results of the byte-based operators are the worst cases for none of the functions.

Table 7 shows the simulation results for $p=75$ and follows the same logic in representing the simulation results as in the Tables 5 and 6 . The byte-based mutation operator exposes a better or equal performance in 79 out of 98 cases when different kind of crossover operators are used. The evarage of genetic searches have better or equal performances of the winner for the functions Ackley, Hyperellipsoid, Multimod, Rastrigin, Schewel, Sphere, and Sumsuares. In none of cases, the byte-based operators demonstrate the worst performance.

The byte-based mutation operator used in simulations is the original mutation operator reported in [28] and increases or decreases the value of a randomly selected byte by 1 . As a result of using a higher cardinality of an alphabet, in some cases, more iterations are needed to achieve the correct byte value by mutations. Alternatively, random mutation can be used instead. As mentioned before, random byte-based mutation operator alters a randomly selected byte in the pre-defined range of $0-255$. Again, as a result of using a higher cardinality of an alphabet, the mutation probability is generally set to higher values, at least in early generations of the genetic search. The byte-based dynamic mutation starts its job for a given mutation probability at generation 0 and reduces the amount of this value by generations and finally sets it to 0 in the final generation and performs the alteration by changing byte values by 1 . Similary, bytebased random dynamic mutation applies the random mutation operator by decreasing the mutation probability by iterations. To reveal the effects of the dynamic mutation probabilities, we perform an other simulation study with the same set of functions but this time for only the byte-based operators. Table 8 and Table 9 summarize the results. In Table 8, the results of the byte-based mutation operators are reported for $p=25$, $p=50$, and $p=75$ for the set of functions. The mutation probabilities are set to $0.10,0.95$, and 0.30 for random, dynamic and random dynamic mutation operators, respectively. Note that the mutation probabilites for dynamic operators are initial and they are reduced by iterations and finally set to 0 at last iteration. The values are average of 250 iterations. It is shown that in Table 8 that the dynamic byte-based operators have better performances for only the Griewank function and the performance does not change in 11 out of 14 cases for $p=25$. For $p=50$, the results are different Dynamic mutation operators have better performances in 5 out of 14 cases and the performance does not change in 8 out of 14 cases. Summarizing these, the performance is better or does not change in 13 out of 14 cases. For the function Levy, original bytebased mutation has a better performance but the difference is small. We have similar results when the number of parameters $p$ is 75 . Dynamic mutation operators have better performances in 8 out of 14 cases. Addition to this, the performance does not change in 5 out of 14 cases. The function Levy has the different status again and the dynamic mutation operators obtain nearly same results with a small and less performance.

Note that there is not a general tumb-of-rule of determining the mutation probability and the initially selected probability can dramatically change the performances. In Table 9 , the results of the same simulation study but this time with the mutation probabilites of $0.1,0.5$, and 0.5 for random, dynamic and random dynamic mutation operators are showed, respectively. It is shown in Table 9 that the smaller dynamic initial mutation probabilities have not better performances on the selected set of test func tions. This results are also affected by the maximum number of iterations. When the 
Table 4: Test Functions Used in Simulations

\begin{tabular}{|c|c|c|}
\hline Function & Definition & Domain \\
\hline Ackley & $\begin{array}{l}20 \exp \left(-0.2 \sqrt{\frac{1}{n} \sum_{i=1}^{n} x_{i}^{2}}\right)- \\
\exp \left(\frac{1}{n} \sum_{i=1}^{n} \cos \left(2 \pi x_{i}\right)\right)+20+e\end{array}$ & $-30 \leq x_{i} \leq 30$ \\
\hline Bohachevksy & $\begin{array}{l}\sum_{i=1}^{n}\left(x_{i}^{2}+2 x_{i+1}^{2}-0.3 \cos \left(3 \pi x_{i}\right)\right. \\
\left.-0.4 \cos \left(4 \pi x_{i+1}\right)+0.7\right)\end{array}$ & $-50 \leq x_{i} \leq 50$ \\
\hline Griewank & $1+\frac{1}{4000} \sum_{i=1}^{n} x_{i}^{2}-\prod_{i=1}^{n} \cos \left(\frac{x_{i}}{\sqrt{i}}\right)$ & $-600 \leq x_{i} \leq 600$ \\
\hline Holzman & $\sum_{i=1}^{n} i x_{i}^{4}$ & $-10 \leq x_{i} \leq 10$ \\
\hline Hyperellipsoid & $\sum_{i=1}^{n} i^{i}+x_{i}^{2}$ & $-5.12 \leq x_{i} \leq 5.12$ \\
\hline Levy & $\begin{array}{l}\sin ^{2}\left(\pi y_{0}\right)+ \\
\sum_{i=0}^{n-2}\left(y_{i}-1\right)^{2}\left(1+10 \sin ^{2}\left(\pi y_{i}+1\right)\right)+ \\
\left(y_{n-1}-1\right)^{2}\left(1+\sin ^{2}\left(2 \pi x_{n-1}\right)\right)\end{array}$ & $\begin{array}{l}y_{i}=1+\frac{x_{i}-1}{4} \\
-10 \leq x_{i} \leq 10\end{array}$ \\
\hline Maxmod & $\max \left(\left|x_{i}\right|\right)$ & $-10 \leq x_{i} \leq 10$ \\
\hline Multimod & $\sum_{i=1}^{n}\left|x_{i}\right| \prod_{i=1}^{n}\left|x_{i}\right|$ & $-10 \leq x_{i} \leq 10$ \\
\hline Rastrigin & $\sum_{i=1}^{n} x_{i}^{2}-10 \cos \left(2 \pi x_{i}\right)+10$ & $-5.12 \leq x_{i} \leq 5.12$ \\
\hline Rosenbrock & $\sum_{i=2}^{n} 100\left(x_{i}-x_{i-1}^{2}\right)^{2}+\left(1+x_{i-1}\right)^{2}$ & $-10 \leq x_{i} \leq 10$ \\
\hline Schaffer & $\begin{array}{l}\sum_{1}^{n-1}\left(\left(x_{i}^{2}+x_{i+1}^{2}\right)^{1 / 4}\right. \\
\left.\sin \left(50\left(x_{i}^{2}+x_{i+1}^{2}\right)^{1 / 10}\right)\right)^{2}+1\end{array}$ & $-100 \leq x_{i} \leq 100$ \\
\hline Schwefel & $\sum_{i=1}^{n}\left\{\sum_{j=1}^{j<i} x_{i}\right\}^{2}$ & $-10 \leq x \leq 10$ \\
\hline Sphere & $\sum_{i=1}^{n} x_{i}^{2}$ & $-10 \leq x_{i} \leq 10$ \\
\hline Sumsquares & $\sum_{i=0}^{n-1} i x_{i}^{2}$ & $-10 \leq x \leq 10$ \\
\hline
\end{tabular}


Table 5: Mean fitness values for $p=25$

\begin{tabular}{|l|l|c|c|c|c|c|c|c|}
\hline Function & Mutation & Byte & SBX & Flat & Arithmetic & BLX & Linear & Unfair \\
\hline \multirow{2}{*}{ Ackley } & Byte & 0 & 0.048 & 1.584 & 1.557 & 1.164 & 0 & 0.008 \\
& Real & 1.294 & 6.278 & 3.61 & 4.259 & 1.651 & 0 & 0 \\
\hline \multirow{2}{*}{ Bohachevsky } & Byte & 0 & 1.438 & 22.124 & 23.042 & 54.459 & 0 & 0.049 \\
& Real & 177.261 & 289.668 & 64.494 & 92.817 & 63.803 & 0 & 0.001 \\
\hline \multirow{2}{*}{ Griewank } & Byte & 0.002 & 0.405 & 1.112 & 1.127 & 1.49 & 0 & 0.011 \\
& Real & 3.876 & 4.43 & 1.607 & 2.008 & 1.582 & 0 & 0.004 \\
\hline \multirow{2}{*}{ Holzman } & Byte & 0 & 0.031 & 0.103 & 0.118 & 25.411 & 0 & 0 \\
& Real & 0 & 52.716 & 1.861 & 2.634 & 85.328 & 0 & 0 \\
\hline \multirow{2}{*}{ Hyperellipsoid } & Byte & 0 & 0.013 & 0.358 & 0.404 & 1.935 & 0 & 0 \\
& Real & 0 & 12.953 & 2.05 & 3.128 & 2.632 & 0 & 0 \\
\hline \multirow{2}{*}{ Levy } & Byte & 2.453 & 1.833 & 0.679 & 0.873 & 0.726 & 0 & 2.001 \\
& Real & 1.271 & 5.062 & 0.453 & 0.689 & 0.754 & 0 & 1.808 \\
\hline \multirow{2}{*}{ Maxmod } & Byte & 0 & 0.799 & 0.475 & 0.516 & 0.566 & 0 & 0.004 \\
& Real & 1.514 & 1.281 & 0.91 & 0.988 & 0.92 & 0 & 0 \\
\hline \multirow{2}{*}{ Multimod } & Byte & 0 & 0 & 0 & 0 & 0 & 0 & 0 \\
& Real & 0 & 0 & 0 & 0 & 0.45 & 0 & 0 \\
\hline \multirow{2}{*}{ Rastrigin } & Byte & 0 & 0.043 & 6.806 & 5.14 & 13.913 & 0 & 0.013 \\
& Real & 0 & 57.656 & 14.109 & 17.189 & 24.749 & 0 & 0 \\
\hline \multirow{2}{*}{ Rosenbrock } & Byte & 24.969 & 54.933 & 59.048 & 61.115 & 580.811 & 0 & 23.393 \\
& Real & 59.324 & 816.942 & 161.611 & 190.214 & 1544.641 & 0 & 23.092 \\
\hline \multirow{2}{*}{ Schaffer } & Byte & 0 & 2.842 & 29.849 & 27.783 & 18.711 & 0.001 & 0.147 \\
& Real & 49.428 & 137.788 & 49.009 & 56.881 & 17.963 & 0.001 & 0.033 \\
\hline \multirow{2}{*}{ Schwefel } & Byte & 0 & 0.817 & 14.167 & 15.643 & 101.578 & 0 & 0.001 \\
& Real & 0 & 655.942 & 80.038 & 129.418 & 147.615 & 0 & 0 \\
\hline \multirow{2}{*}{ Sphere } & Byte & 0 & 0.005 & 0.126 & 0.141 & 0.533 & 0 & 0 \\
& Real & 0 & 3.851 & 0.679 & 1.073 & 0.661 & 0 & 0 \\
\hline \multirow{2}{*}{ Sumsquares } & Byte & 0 & 0.048 & 1.423 & 1.526 & 7.392 & 0 & 0 \\
& Real & 0 & 51.571 & 7.18 & 11.572 & 10.233 & 0 & 0 \\
\hline
\end{tabular}


Table 6: Mean fitness values for $p=50$

\begin{tabular}{|l|l|c|c|c|c|c|c|c|}
\hline Function & Mutation & Byte & SBX & Flat & Arithmetic & BLX & Linear & Unfair \\
\hline \multirow{2}{*}{ Ackley } & Byte & 0 & 1.894 & 3.797 & 3.857 & 4.094 & 0 & 0.102 \\
& Real & 6.121 & 6.894 & 5.919 & 6.643 & 4.272 & 0 & 0 \\
\hline \multirow{2}{*}{ Bohachevsky } & Byte & 0 & 128.977 & 173.791 & 198.861 & 634.252 & 0 & 0.531 \\
& Real & 1715.242 & 862.481 & 603.124 & 792.846 & 535.86 & 0 & 0.003 \\
\hline \multirow{2}{*}{ Griewank } & Byte & 0.053 & 2.431 & 2.749 & 2.999 & 8.232 & 0 & 0.13 \\
& Real & 22.532 & 11.236 & 7.919 & 10.358 & 7.183 & 0 & 0.008 \\
\hline \multirow{2}{*}{ Holzman } & Byte & 0 & 80.115 & 9.939 & 12.707 & 5673.944 & 0 & 0.001 \\
& Real & 452.34 & 413.445 & 116.911 & 155.7 & 31541.24 & 0 & 0.001 \\
\hline \multirow{2}{*}{ Hyperellipsoid } & Byte & 0 & 8.367 & 10.493 & 12.735 & 53.307 & 0 & 0.004 \\
& Real & 0.058 & 81.879 & 45.693 & 59.439 & 49.24 & 0 & 0 \\
\hline \multirow{2}{*}{ Levy } & Byte & 4.861 & 5.284 & 2.379 & 2.752 & 5.373 & 0 & 4.354 \\
& Real & 4.412 & 53.691 & 2.687 & 3.283 & 8.74 & 0 & 4.043 \\
\hline \multirow{2}{*}{ Maxmod } & Byte & 1.518 & 1.305 & 1.053 & 1.102 & 2.308 & 0 & 0.043 \\
& Real & 4.233 & 1.656 & 1.608 & 1.626 & 4.595 & 0 & 0 \\
\hline \multirow{2}{*}{ Multimod } & Byte & 0 & 0 & 0 & 0 & 0 & 0 & 0 \\
& Real & 0 & 0 & 0 & 0 & $>1000000$ & 0 & 0 \\
\hline \multirow{2}{*}{ Rastrigin } & Byte & 0 & 8.502 & 55.884 & 49.377 & 106.133 & 0 & 0.304 \\
& Real & 0 & 281.093 & 62.923 & 75.051 & 113.491 & 0 & 0 \\
\hline \multirow{2}{*}{ Rosenbrock } & Byte & 53.352 & 881.317 & 367.65 & 396.98 & 29782.03 & 0 & 48.796 \\
& Real & 3772.472 & 2548.67 & 1369.221 & 1631.746 & 150504.3 & 0 & 48.539 \\
\hline \multirow{2}{*}{ Schaffer } & Byte & 0.113 & 41.752 & 112.384 & 112.842 & 90.65 & 0.002 & 0.711 \\
& Real & 166.896 & 391.971 & 142.656 & 154.521 & 81.009 & 0 & 0.055 \\
\hline \multirow{2}{*}{ Schwefel } & Byte & 0 & 591.174 & 837.454 & 990.897 & 5605.509 & 0 & 0.042 \\
& Real & 150.019 & 9187.583 & 4083.564 & 6103.616 & 5623.049 & 0 & 0.002 \\
\hline \multirow{2}{*}{ Sphere } & Byte & 0 & 1.485 & 1.916 & 2.191 & 8.015 & 0 & 0.01 \\
& Real & 0.187 & 11.29 & 7.696 & 10.358 & 6.807 & 0 & 0 \\
\hline \multirow{2}{*}{ Sumsquares } & Byte & 0 & 32.996 & 40.417 & 46.195 & 208.287 & 0 & 0.013 \\
& Real & 4.723 & 299.023 & 169.286 & 234.566 & 188.943 & 0 & 0 \\
\hline
\end{tabular}


Table 7: Mean fitness values for $p=75$

\begin{tabular}{|l|l|c|c|c|c|c|c|c|}
\hline Function & Mutation & Byte & SBX & Flat & Arithmetic & BLX & Linear & Unfair \\
\hline \multirow{2}{*}{ Ackley } & Byte & 0 & 4.309 & 5.184 & 5.463 & 6.202 & 0 & 0.195 \\
& Real & 8.912 & 6.802 & 7.307 & 7.775 & 6.544 & 0 & 0 \\
\hline \multirow{2}{*}{ Bohachevsky } & Byte & 5.116 & 747.24 & 589.389 & 697.32 & 2360.041 & 0 & 1.348 \\
& Real & 511.868 & 1308.588 & 1690.507 & 2049.271 & 2178.298 & 0 & 0.009 \\
\hline \multirow{2}{*}{ Griewank } & Byte & 0.9 & 9.409 & 7.417 & 8.524 & 28.747 & 0 & 0.277 \\
& Real & 63.822 & 16.846 & 20.864 & 25.33 & 26.622 & 0 & 0.013 \\
\hline \multirow{2}{*}{ Holzman } & Byte & 0.249 & 523.824 & 89.572 & 115.241 & 60087.81 & 0 & 0.014 \\
& Real & 9394.847 & 1179.416 & 874.926 & 920.62 & 447819.4 & 0 & 0.018 \\
\hline \multirow{2}{*}{ Hyperellipsoid } & Byte & 0 & 76.486 & 56.233 & 68.987 & 289.971 & 0 & 0.019 \\
& Real & 0.872 & 184.472 & 197.084 & 239.135 & 289.815 & 0 & 0 \\
\hline \multirow{2}{*}{ Levy } & Byte & 7.178 & 16.63 & 4.484 & 5.135 & 20.172 & 0 & 6.679 \\
& Real & 9.835 & 128.98 & 6.378 & 7.606 & 41.243 & 0 & 6.269 \\
\hline \multirow{2}{*}{ Maxmod } & Byte & 3.931 & 1.604 & 1.499 & 1.507 & 4.108 & 0 & 0.114 \\
& Real & 5.875 & 1.871 & 2.007 & 1.968 & 7.12 & 0 & 0.001 \\
\hline \multirow{2}{*}{ Multimod } & Byte & 0 & 0 & 0 & 0 & 0 & 0 & 0 \\
& Real & 0 & 0 & 0 & 0 & $>1000000$ & 0 & 0 \\
\hline \multirow{2}{*}{ Rastrigin } & Byte & 0 & 54.623 & 144.001 & 135.201 & 275.679 & 0 & 0.831 \\
& Real & 0.049 & 585.346 & 141.157 & 171.444 & 290.118 & 0 & 0.027 \\
\hline \multirow{2}{*}{ Rosenbrock } & Byte & 109.211 & 3182.507 & 1150.206 & 1283.993 & 213157.8 & 0 & 77.112 \\
& Real & 31932.39 & 4260.689 & 4576.93 & 4772.071 & 1453959 & 0 & 74.04 \\
\hline \multirow{2}{*}{ Schaffer } & Byte & 0.86 & 129.83 & 214.884 & 221.946 & 203.911 & 0.003 & 1.058 \\
& Real & 311.443 & 647.206 & 246.625 & 259.942 & 206.619 & 0 & 0.069 \\
\hline \multirow{2}{*}{ Schwefel } & Byte & 0 & 9430.678 & 7382.519 & 8708.908 & 44800.49 & 0 & 0.285 \\
& Real & 8449.909 & 33311.05 & 28855.81 & 37370.13 & 46206.6 & 0 & 0.011 \\
\hline \multirow{2}{*}{ Sphere } & Byte & 0 & 9.33 & 7.159 & 8.639 & 30.998 & 0 & 0.043 \\
& Real & 5.48 & 17.949 & 22.211 & 26.552 & 28.889 & 0 & 0 \\
\hline \multirow{2}{*}{ Sumsquares } & Byte & 0 & 288.37 & 221.715 & 260.394 & 1135.356 & 0 & 0.051 \\
& Real & 237.674 & 717.93 & 739.906 & 907.269 & 1120.843 & 0 & 0 \\
\hline
\end{tabular}


Table 8: pmutation $=0.1,0.95,0.3$, respectively

\begin{tabular}{|c|c|c|c|c|}
\hline$p$ & Functions & random & dynamic & random_dynamic \\
\hline \multirow{14}{*}{25} & Ackley & 0 & 0 & 0 \\
\hline & Bohachevsky & 0 & 0 & 0 \\
\hline & Griewank & 0.007 & 0 & 0 \\
\hline & Holzman & 0 & 0 & 0 \\
\hline & Hyperellipsoid & 0 & 0 & 0 \\
\hline & Levy & 1.306 & 2.007 & 1.426 \\
\hline & Maxmod & 0 & 0 & 0 \\
\hline & Multimod & 0 & 0 & 0 \\
\hline & Rastrigin & 0 & 0 & 0 \\
\hline & Rosenbrock & 23.674 & 23.867 & 23.611 \\
\hline & Schaffer & 0 & 0 & 0 \\
\hline & Schwefel & 0 & 0 & 0 \\
\hline & Sphere & 0 & 0 & 0 \\
\hline & Sumsquares & 0 & 0 & 0 \\
\hline \multirow{14}{*}{50} & Ackley & 0 & 0 & 0 \\
\hline & Bohachevsky & 2.029 & 0 & 0 \\
\hline & Griewank & 0.795 & 0 & 0.003 \\
\hline & Holzman & 0 & 0 & 0 \\
\hline & Hyperellipsoid & 0 & 0 & 0 \\
\hline & Levy & 3.713 & 4.369 & 3.77 \\
\hline & Maxmod & 1.555 & 0 & 0 \\
\hline & Multimod & 0 & 0 & 0 \\
\hline & Rastrigin & 0 & 0 & 0 \\
\hline & Rosenbrock & 54.439 & 48.939 & 48.826 \\
\hline & Schaffer & 0.001 & 0 & 0 \\
\hline & Schwefel & 0 & 0 & 0 \\
\hline & Sphere & 0 & 0 & 0 \\
\hline & Sumsquares & 0 & 0 & 0 \\
\hline \multirow{14}{*}{75} & Ackley & 0.278 & 0 & 0 \\
\hline & Bohachevsky & 181.757 & 0 & 0.057 \\
\hline & Griewank & 2.232 & 0 & 0.271 \\
\hline & Holzman & 0.684 & 0 & 0 \\
\hline & Hyperellipsoid & 0 & 0 & 0 \\
\hline & Levy & 6.21 & 6.791 & 6.239 \\
\hline & Maxmod & 4.253 & 0 & 0.003 \\
\hline & Multimod & 0 & 0 & 0 \\
\hline & Rastrigin & 0 & 0 & 0 \\
\hline & Rosenbrock & 97.479 & 73.973 & 73.911 \\
\hline & Schaffer & 0.385 & 0 & 0 \\
\hline & Schwefel & 0.351 & 0 & 0.003 \\
\hline & Sphere & 0 & 0 & 0 \\
\hline & Sumsquares & 0 & 0 & 0 \\
\hline
\end{tabular}

maximum number of iterations is set to a higher integer, the amount of decrease on the mutation probability is small and the effect of the mutation operator is higher at the early stages of the genetic search. However, as the maximum number of generations is small, the decrease on the mutation probability increases and the algorithm suddenly enters a fine-tuning stage. Finally, the fine-tuned solution is probably a local optimum because the algorithm may not discover the different parts of the search space. Determining a good start for the dynamic mutation probability can be the subject of a further research

\section{Conclusion}

Machine-coded genetic operators are applied on the byte representation of candidate solutions on the computer memory. Byte-based crossover operators recombines real vectors to generate offspring on a way similar to their binary counterpars using the geno-type, namely, bytes. The original byte-based mutation operator also mimics its binary counterpart by altering a single byte by \pm 1 . In this paper, we introduce the random byte-based mutation operator in which a single byte is altered randomly in the range of a byte in computer memory. We also introduce the dynamic byte-based mutation operators in which the mutation probability is dynamically decreased by iterations to perform the tasks of exploration and exploitation in a more efficient way. Byte-based dynamic mutation operators search the different areas of the search space when the mutation probability is high and the task is turned into performing a local fine-tuning by decreasing the mutation probability by iterations. Since the effect of the byte-based mutation operators depends on the location of the mutated byte, a long jump or a local fine-tuning can be carried out without setting a user-defined option such as 
a parameter of a probability density function. When a moderate size of population is used and the maximum number of iterations is enough to converge, byte-based operators obtain very precise results even with the decision variables with wider domain because of the constant-sized and compact representation of real numbers in range of roughly $\left(-10^{308}, 10^{308}\right)$ with 8-bytes long double type.

A simulation study is performed on a set of well-known test functions that are used for comparing performances of evolutionary optimization algorithms in recent studies. Simulations are ran on the R system with GA and mcga packages installed. The package mcga implements the byte-based operators and freely avaliable for downloading Results of the simulation study show that the byte-based operators performs well when they are combined for reproduction tasks. The performance increases when the initia mutation probability is set to a higher value and then dynamically decreased, and finally it is set to 0 at last generation.

The developed byte-based operators are based on the double type which is 8 bytes long. Alternatively 4 bytes long float and 16 bytes long long double data types can be used for representing floating-point numbers. Performances of developed operators on these data types are not investigated and this can be a subject of a further study.

\section{References}

[1] Ernesto P Adorio and U Diliman. Mvf-multivariate test functions library in c for unconstrained global optimization. Quezon City, Metro Manila, Philippines, 2005

[2] M Montaz Ali, Charoenchai Khompatraporn, and Zelda B Zabinsky. A numerical evaluation of several stochastic algorithms on selected continuous global optimization test problems. Journal of Global Optimization, 31(4):635-672, 2005.

[3] Rachid Chelouah and Patrick Siarry. Tabu search applied to global optimization. European journal of operational research, 123(2):256$270,2000$.

[4] Sung-Bae Cho and Joo-Young Lee. A human-oriented image retrieval system using interactive genetic algorithm. Systems, Man and Cybernetics, Part A: systems and humans, IEEE Transactions on, 32(3):452458,2002

[5] Kalyanmoy Deb. Multi-objective optimization using evolutionary algorithms, volume 16. John Wiley \& Sons, 2001

[6] Kalyanmoy Deb and Ram Bhushan Agrawal. Simulated binary crossover for continuous search space. Complex systems, 9(2):115148,1995

[7] Kalyanmoy Deb and A Kumar. Real-coded genetic algorithms with simulated-binary crossover: Studies on multi-modal and multiobjective problems. Complex systems, 9(6):431-454, 1995.

[8] Larry J Eshelman. chapter real-coded genetic algorithms and intervalschemata. Foundations of genetic algorithms, 2:187-202, 1993.

[9] David Goldberg. What every computer scientist should know about floating-point arithmetic. ACM Computing Surveys (CSUR), 23(1):548, 1991.

[10] David E. Goldberg. Genetic Algorithms in Search, Optimization and Machine Learning. Addison-Wesley Longman Publishing Co., Inc., Boston, MA, USA, 1st edition, 1989.

[11] John J Grefenstette. Optimization of control parameters for genetic algorithms. Systems, Man and Cybernetics, IEEE Transactions on 16(1):122-128, 1986.

[12] Nikolaus Hansen and Stefan Kern. Evaluating the cma evolution strategy on multimodal test functions. In Parallel problem solving from nature-PPSN VIII, pages 282-291. Springer, 2004.

[13] Francisco Herrera and Manuel Lozano. Gradual distributed real-coded genetic algorithms. Evolutionary Computation, IEEE Transactions on 4(1):43-63, 2000.

[14] Francisco Herrera, Manuel Lozano, and Ana M Sánchez. A taxonomy for the crossover operator for real-coded genetic algorithms: An experimental study. International Journal of Intelligent Systems, 18(3):309_ 338,2003

[15] Francisco Herrera, Manuel Lozano, and Jose L. Verdegay. Tackling real-coded genetic algorithms: Operators and tools for behavioural analysis. Artificial intelligence review, 12(4):265-319, 1998.

[16] John H Holland. Adaptation in Natural and Artificial Systems: An Introductory Analysis with Applications to Biology, Control, and Artificial Intelligence. U Michigan Press, 1975.

[17] Hee-Su Kim and Sung-Bae Cho. Application of interactive genetic algorithm to fashion design. Engineering applications of artificial intelligence, 13(6):635-644, 2000.

[18] Alex Kosorukoff. Human based genetic algorithm. In Systems, Man, and Cybernetics, 2001 IEEE International Conference on, volume 5 , pages 3464-3469. IEEE, 2001.

[19] Fernando G Lobo and David E Goldberg. The parameter-less genetic algorithm in practice. Information Sciences, 167(1):217-232, 2004

[20] Zbigniew Michalewicz. Genetic algorithms + data structures = evolution programs. Springer Science \& Business Media, 2013

[21] Tatsuya Nomura and Tsutomu Miyoshi. Numerical coding and unfair average crossover in ga for fuzzy rule extraction in dynamic environments. In Fuzzy Logic, Neural Networks, and Evolutionary Computation, pages 55-72. Springer, 1995.

[22] Anne L Olsen. Penalty functions and the knapsack problem. In Evolutionary Computation, 1994. IEEE World Congress on Computational Intelligence., Proceedings of the First IEEE Conference on, pages 554558. IEEE, 1994.
[23] Petr Pospichal, Josef Schwarz, and Jiri Jaros. Parallel genetic algorithm solving $0 / 1$ knapsack problem running on the gpu. In 16th International Conference on Soft Computing MENDEL, volume 2010, pages $64-70,2010$

[24] R Core Team. R: A Language and Environment for Statistical Computing. R Foundation for Statistical Computing, Vienna, Austria, 2016.

[25] Nicholas J Radcliffe. Equivalence class analysis of genetic algorithms Complex systems, 5(2):183-205, 1991.

[26] Shahryar Rahnamayan, Hamid R Tizhoosh, and Magdy Salama Opposition-based differential evolution. Evolutionary Computation, IEEE Transactions on, 12(1):64-79, 2008

[27] Shahryar Rahnamayan, Hamid R Tizhoosh, and Magdy MA Salama. A novel population initialization method for accelerating evolutionary algorithms. Computers \& Mathematics with Applications, 53(10):16051614, 2007.

[28] Mehmet Hakan Satman. Machine coded genetic algorithms for real parameter optimization problems. Gazi University Journal of Science, 26(1):85-95, 2013

[29] Mehmet Hakan Satman and Emre Akadal. Arima forecasting as a genetic inheritance operator in floating-point genetic algorithms. Journal of Mathematical and Computational Science, 6(3):360, 2016.

[30] Luca Scrucca. Ga: A package for genetic algorithms in r. Journal of Statistical Software, 53(1):1-37, 2013.

[31] D. Stevenson. A proposed standard for binary floating-point arithmetic. Computer, 14(3):51-62, March 1981.

[32] Rainer Storn and Kenneth Price. Differential evolution-a simple and efficient heuristic for global optimization over continuous spaces. Jour nal of global optimization, 11(4):341-359, 1997.

[33] Aram Ter-Sarkisov and Stephen Marsland. K-bit-swap: a new operator for real-coded evolutionary algorithms. Soft Computing, pages 1-10, 2016

[34] Jakob Vesterstrøm and Rene Thomsen. A comparative study of differential evolution, particle swarm optimization, and evolutionary algorithms on numerical benchmark problems. In Evolutionary Computation, 2004. CEC2004. Congress on, volume 2, pages 1980-1987. IEEE, 2004.

[35] Alden H Wright. Genetic algorithms for real parameter optimization. Foundations of genetic algorithms, 1:205-218, 1991. 